\title{
Choledocholithiasis in acute calculous cholecystitis: guidelines and beyond
}

\author{
Siddhartha Reddy, Nitin Jagtap, Rakesh Kalapala, Mohan Ramchandani, Sundeep Lakhtakia, \\ Jahangeer Basha, Zaheer Nabi, Arun Karyampudi, Radhika Chavan, Manu Tandan, Rajesh Gupta, \\ D. Nageshwar Reddy
}

Asian Institute of Gastroenterology, Hyderabad, India

Abstract

\begin{abstract}
Background Acute calculous cholecystitis (ACC) is the most frequent complication of gallstones requiring cholecystectomy. These patients may have coexisting choledocholithiasis. We aimed to evaluate the role of current guidelines for choledocholithiasis in patients with ACC.
\end{abstract}

\begin{abstract}
Methods In this retrospective study, we included all patients diagnosed with ACC between December 2018 and May 2019. These patients were substratified according to the guidelines of the American and European Societies of Gastrointestinal Endoscopy (ASGE and ESGE) as having high, intermediate, or low likelihood of choledocholithiasis, and the diagnostic performance was measured. Binomial logistic regression analysis was applied to ascertain independent risk factors for choledocholithiasis.

Results A total of 173 patients with ACC, mean age ( \pm standard deviation) $49.89 \pm 15.74$ years and $60.1 \%$ male, were included. Sixty-three $(36.4 \%)$ had confirmed choledocholithiasis. ASGE high likelihood criteria had sensitivity and specificity of $61.9 \%$ (95\% confidence interval [CI] 48.8-73.9) and $83.4 \%$ (95\%CI 75.4-90.0) for predicting choledocholithiasis. ESGE high likelihood criteria had sensitivity and specificity of $49.2 \%$ (95\%CI 36.4-62.1) and 87.3\% (95\%CI 79.6-92.9). On logistic regression analysis, an alkaline phosphatase level above the upper limit of normal $(\mathrm{P}=0.003$; odds ratio [OR] 4.26, 95\%CI 1.66-10.96) and a dilated common bile duct on ultrasound ( $\mathrm{P}=0.001$; OR 9.97, 95\%CI 4.65-21.36) were independent positive predictors for choledocholithiasis, while acute biliary pancreatitis was an independent negative predictor $(\mathrm{P}=0.030$; OR $0.36,95 \% \mathrm{CI} 0.14-0.91)$.
\end{abstract}

Conclusions The performance of the ASGE and ESGE guidelines' risk stratification criteria is inadequate in patients with ACC. We suggest the utilization of a separate predictive model for suspected choledocholithiasis in these patients.

Keywords Acute calculous cholecystitis, choledocholithiasis, predictors, gallstones, risk

Ann Gastroenterol 2021; 34 (1): 1-6

\section{Introduction}

Acute calculous cholecystitis (ACC) is the most frequent complication of gallstones, accounting for $14-30 \%$ of

Department of Gastroenterology, Asian Institute of Gastroenterology, Hyderabad, India (Siddhartha Reddy, Nitin Jagtap, Rakesh Kalapala, Mohan Ramchandani, Sundeep Lakhtakia, Jahangeer Basha, Zaheer Nabi, Arun Karyampudi, Radhika Chavan, Manu Tandan, Rajesh Gupta, D. Nageshwar Reddy)

Conflict of Interest: None

Correspondence to: Dr. Nitin Jagtap, Consultant Gastroenterology, Asian Institute of Gastroenterology, Hyderabad, India, e-mail: docnits13@gmail.com

Received 31 May 2020; accepted 9 September 2020; published online 7 December 2020

DOI: https://doi.org/10.20524/aog.2020.0562 cholecystectomies [1,2]. Patients with symptomatic gallstones carry more risk for the development of ACC than patients without symptoms [3]. Symptomatic gallstones also increase the risk for other complications, such as gallstone pancreatitis and choledocholithiasis. ACC is diagnosed according to the Tokyo guidelines with a combination of clinical, laboratory, and imaging findings [4,5]. Initial investigations include liver function tests (LFTs), hemogram and abdominal ultrasound (US). A dilated common bile duct (CBD) on US and/or alerted LFTs should raise the suspicion for choledocholithiasis. Coexisting choledocholithiasis needs to be recognized early as it may lead to jaundice and/or cholangitis, the incidence of which is reported to be $13.7 \%$ (range $3.36-25 \%$ ) in a metaanalysis [6].

The American and European Societies of Gastrointestinal Endoscopy (ASGE and ESGE) have recently updated their guidelinesforthemanagementofcholedocholithiasis [7,8]. These guidelines stratify patients with suspected choledocholithiasis 
into high, intermediate and low likelihood groups, depending on the presence of clinical cholangitis, LFTs, and US findings (Table 1). However, these guidelines do not mention their clinical application in the presence of ACC. ACC per se is associated with an alteration of LFTs, irrespective of choledocholithiasis, which may decrease the clinical utility of these guidelines in this subset of patients. In the current study, we aimed to evaluate the diagnostic performance of the ASGE and ESGE guidelines for the prediction of choledocholithiasis in patients with ACC.

\section{Patients and methods}

Institutional Review Board approval was obtained. In this single-center retrospective study of prospectively maintained data we included all consecutive patients diagnosed with ACC between December 2018 and May 2019. ACC was diagnosed as per Tokyo guidelines $[5,9]$. Patients with underlying liver disease, alcohol consumption, prior biliary surgery, prior endoscopic retrograde cholangiopancreatography (ERCP), biliary stricture, or primary sclerosing cholangitis were excluded. All included patients were stratified as having a high, intermediate or low likelihood of choledocholithiasis, as per ASGE and ESGE criteria. Coexisting acute pancreatitis was diagnosed according to the revised Atlanta classification [10]. The diagnosis of acute cholangitis in the presence of ACC is clinically difficult: we diagnosed acute cholangitis in patients who had confirmed CBD stones on ERCP and clinical improvement after CBD stone clearance or biliary stenting.

The data were collected in a standardized data collection sheet, which included demographic data such as age, sex, LFTs at admission, serum amylase or lipase whenever indicated, US findings including presence of CBD dilatation, CBD stone/ sludge, signs of ACC, and pancreatitis. For confirmation of CBD stones, details of endoscopic procedures endoscopic US and ERCP, and the radiological procedure magnetic resonance cholangiopancreatography (MRCP) were noted whenever they were performed. Patients in whom CBD stones were not detected during an initial presentation but were detected within 6 months of follow up were considered as having a missed CBD stone.

The categorical data were presented as proportions. The continuous data were presented as mean and standard deviation (SD). The diagnostic performance of the ASGE and ESGE criteria was presented as sensitivity, specificity, positive and negative predictive value, likelihood ratio + and - along with respective $95 \%$ confidence interval (CI). Clopper-Pearson
CIs were calculated for sensitivity and specificity. The log method was used to calculate the CIs for the likelihood ratio, and standard logit was used to obtain CIs for the predictive values. Binominal logistic regression analysis using a forward conditional method was carried out to ascertain independent predictors of $\mathrm{CBD}$ stones among the variables that were significant in univariate analysis. Acute cholangitis was not included in the analysis, as it was diagnosed only in patients with confirmed CBD stones. A P-value $<0.05$ was considered as statistical significance. SPSS version (IBM Corp., Armonk, New York, USA) and MedCalc version 19.1.3 (MedCalc Software bv, Ostend, Belgium) were used for the statistical analysis.

\section{Results}

A total of 173 patients with ACC, mean age $( \pm S D)$ $49.89 \pm 15.74$ years and $60.1 \%$ male, were included in the analysis; of these $43(24.9 \%)$ had coexisting acute pancreatitis and 15 (8.67\%) had cholangitis. There were 63 (36.4\%) confirmed cases of choledocholithiasis. Other baseline characteristics of the study population are summarized in Table 2.

After analysis of clinical, laboratory and US findings, patients were stratified according to the ASGE criteria as follows: high likelihood of choledocholithiasis 57 (32.9\%), intermediate likelihood 105 (60.7\%) and low likelihood 11 (6.4\%); and according to ESGE criteria as follows: 45 (26\%) high, 109 (63\%) intermediate and 19 (11\%) low likelihood. Of the patients who satisfied the ASGE and ESGE high likelihood criteria, 39/57 (68.4\%) and 31/45 (68.9\%) had choledocholithiasis respectively. In the ASGE and ESGE intermediate likelihood group, 24/105 (22.85\%) and 31/109 (28.44\%) had choledocholithiasis, respectively. Only one patient in the ESGE low likelihood group had choledocholithiasis. The diagnostic performance of the ASGE and ESGE guidelines is summarized in Table 3. ASGE high likelihood criteria had sensitivity and specificity of $61.9 \%$ (95\%CI $48.8-73.9$ ) and $83.4 \%$ (95\%CI 75.4-90.0) for predicting choledocholithiasis. ESGE high likelihood criteria had sensitivity and specificity of 49.2\% (95\%CI 36.4-62.1) and specificity $87.3 \%$ (95\%CI 79.6-92.9). The risk of undergoing diagnostic ERCP according to the high likelihood ESGE and ASGE criteria is $31.1 \%$ and $31.6 \%$, respectively.

On univariate analysis, we found that elevated bilirubin and alkaline phosphatase (ALP) levels, dilated CBD on US, CBD stone on US, and acute biliary pancreatitis were significant risk factors for choledocholithiasis in patients with ACC $(\mathrm{P}<0.05)$. On logistic regression analysis using a forward conditional

Table 1 American and European Societies of Gastrointestinal Endoscopy risk stratification criteria

\begin{tabular}{llll}
\hline Society & High likelihood & Intermediate likelihood & Low Likelihood \\
\hline ESGE & Cholangitis or CBD stone on US & $\begin{array}{l}\text { Abnormal LFT and/or CBD } \\
\text { dilatation on US }\end{array}$ & Normal LFT and US \\
ASGE & & Age $>55$ years & \\
& Combination of Serum Bilirubin $>4 \mathrm{mg} / \mathrm{dL}$ and CBD dilatation on US & & \\
\hline
\end{tabular}

ESGE, European Society of Gastrointestinal Endoscopy; ASGE, American Society of Gastrointestinal Endoscopy; US, ultrasound; CBD, common bile duct; LFT, liver function test 
method, ALP elevated above the upper limit of normal $(\mathrm{P}=0.003$; odds ratio [OR] 4.26, 95\%CI 1.66-10.96) and dilated CBD on US ( $\mathrm{P}=0.001$; OR 9.97, 95\%CI 4.65-21.36) were independent positive predictors for choledocholithiasis, while acute biliary pancreatitis was an independent negative predictor $(\mathrm{P}=0.030$; OR $0.36,95 \%$ CI $0.14-0.91$ ) (Table 4 ). The predictive score was calculated by allotting +1 score for each positive predictor and -1 for each negative predictor, giving a range from -1 to +2 . Using $\geq 1$ as cutoff, sensitivity and specificity for predicting choledocholithiasis were 95.24\% (95\%CI 86.71-99.01) and $51.82 \%$ (95\%CI $42.09-61.45$ ). When the score was -1 or $0,95 \%$ of patients did not have choledocholithiasis. When the score was $1,28 / 68(41.17 \%)$ had choledocholithiasis, and when the score was 2, 32/45 (71.11\%) had choledocholithiasis.

\section{Discussion}

ACC is the most common complication of symptomatic gallstones [1]. In a significant proportion of patients with ACC other complications, such as choledocholithiasis, cholangitis, or biliary pancreatitis, may coexist [4]. Current guidelines for the management of choledocholithiasis do not separately

Table 2 Baseline characteristics of the study population $(n=173)$

\begin{tabular}{lc} 
Parameter & Mean \pm SD \\
\hline Age in years & $49.89 \pm 15.74$ \\
Sex, male (\%) & $104(60.1 \%)$ \\
Acute biliary pancreatitis, n (\%) & $43(24.9 \%)$ \\
Dilated CBD on US, n (\%) & $72(41.6 \%)$ \\
CBD stone/sludge on US, n (\%) & $36(20.8 \%)$ \\
Serum bilirubin & $3.93 \pm 5.12$ \\
ALT & $101.09 \pm 128.58$ \\
ALP & $212.01 \pm 149.72$ \\
\hline Confirmed choledocholithiasis, n (\%) & $63(36.4 \%)$ \\
\hline
\end{tabular}

SD, standard deviation; CBD, common bile duct; US, ultrasonography; $A L T$, alanine aminotransferase; $A L P$, alkaline phosphatase address the evaluation and management of coexisting ACC and choledocholithiasis $[7,8]$.

In the current study, we evaluated the diagnostic utility of ASGE and ESGE algorithms for diagnosing choledocholithiasis in patients with ACC. The sensitivity and specificity of the ASGE high likelihood criteria were $61.9 \%$ and $83.4 \%$, while the ESGE high likelihood criteria's sensitivity and specificity were $49.2 \%$ and $87.3 \%$, respectively. The sensitivity and specificity of the ASGE high likelihood criteria were $74.64 \%$ and $96.87 \%$, respectively in all patients with symptomatic cholelithiasis who had suspected cholelithiasis, irrespective of the presence of ACC, while for the ESGE high probability criteria the sensitivity and specificity were $74.28 \%$ and $98.96 \%$, respectively [11]. Thus, the sensitivity and specificity of current guidelines to predict choledocholithiasis appear suboptimal in the presence of ACC compared to the overall population. However, the diagnostic performance of these guidelines in the intermediate likelihood group of patients with ACC appeared to be comparable or slightly superior to that in the overall population [11]. These differences underline the need to re-stratify patients who have ACC with suspected choledocholithiasis. This difference could be explained by frequent alterations of LFTs due to nonspecific hepatitis in ACC and significant pericholecystic edema and/or inflammatory changes along the cystic duct and CBD, irrespective of choledocholithiasis [12-15]. This study showed that there is a need to develop other predictive clinical tools to rule out choledocholithiasis on patients with ACC.

We evaluated independent predictive factors for choledocholithiasis using logistic regression analysis with a forward conditional method. Elevated ALP above the upper limit of normal (OR 4.26, 95\%CI 1.66-10.96) and dilated CBD on US (OR 9.97, 95\%CI 4.65-21.36) were independent positive predictors, whereas coexisting acute biliary pancreatitis was an independent negative predictor (OR 0.36, 95\%CI 0.14-0.91). Previous studies have shown good sensitivity and specificity of raised ALP and $\gamma$-glutamyl transpeptidase, and dilated CBD on US, for predicting choledocholithiasis [15-17]. Acute biliary pancreatitis is considered to be a negative predictor for choledocholithiasis, demonstrated by various studies in the past $[18,19]$. This is possibly because acute biliary pancreatitis is caused by small stones or sludge that may pass the papilla

Table 3 Diagnostic performance of ESGE and ASGE criteria for prediction of choledocholithiasis in acute calculous cholecystitis

\begin{tabular}{|c|c|c|c|c|c|c|c|c|}
\hline \multirow[t]{2}{*}{ Society } & \multirow{2}{*}{$\begin{array}{l}\text { True Positive/ } \\
\text { Total Positive }\end{array}$} & \multirow{2}{*}{$\begin{array}{l}\text { True Negative/ } \\
\text { Total Negative }\end{array}$} & Sensitivity & Specificity & PPV & NPV & $\mathrm{LR}+$ & LR- \\
\hline & & & \multicolumn{6}{|c|}{$\%(95 \%$ confidence interval) } \\
\hline ASGE High & $39 / 57$ & $92 / 116$ & $\begin{array}{c}61.9 \\
(48.8-73.9)\end{array}$ & $\begin{array}{c}83.4 \\
(75.4-90.0)\end{array}$ & $\begin{array}{c}68.4 \\
(57.7-77.5)\end{array}$ & $\begin{array}{c}79.3 \\
(73.5-84.2)\end{array}$ & $\begin{array}{c}3.78 \\
(2.38-6.02)\end{array}$ & $\begin{array}{c}0.46 \\
(0.33-0.63)\end{array}$ \\
\hline ASGE Intermediate & $24 / 105$ & $29 / 68$ & $\begin{array}{c}38.1 \\
(26.2-51.2)\end{array}$ & $\begin{array}{c}26.4 \\
(18.4-35.6)\end{array}$ & $\begin{array}{c}22.9 \\
(17.5-29.3)\end{array}$ & $\begin{array}{c}42.7 \\
(33.9-51.8)\end{array}$ & $\begin{array}{c}0.52 \\
(0.37-0.72)\end{array}$ & $\begin{array}{c}2.35 \\
(1.63-3.39)\end{array}$ \\
\hline ESGE High & $31 / 45$ & $96 / 128$ & $\begin{array}{c}49.2 \\
(36.4-62.1)\end{array}$ & $\begin{array}{c}87.3 \\
(79.6-92.9)\end{array}$ & $\begin{array}{c}68.9 \\
(56.1-79.4)\end{array}$ & $\begin{array}{c}75.0 \\
69.9-79.4)\end{array}$ & $\begin{array}{c}3.87 \\
(2.23-6.70)\end{array}$ & $\begin{array}{c}0.58 \\
(0.45-0.75)\end{array}$ \\
\hline ESGE intermediate & $31 / 109$ & $32 / 64$ & $\begin{array}{c}49.2 \\
(36.4-62.1)\end{array}$ & $\begin{array}{c}29.1 \\
(20.8-38.5)\end{array}$ & $\begin{array}{c}28.4 \\
(23.1-34.4)\end{array}$ & $\begin{array}{c}50.0 \\
(40.6-59.4)\end{array}$ & $\begin{array}{c}0.69 \\
(0.53-0.92)\end{array}$ & $\begin{array}{c}1.75 \\
(1.19-2.55)\end{array}$ \\
\hline
\end{tabular}

ASGE, American Society of Gastrointestinal Endoscopy; ESGE, European Society of Gastrointestinal Endoscopy; PPV, positive predictive value; NPV, negative predictive value; $L R+$, likelihood ratio plus; $L R$-, likelihood ratio minus 
Table 4 Univariate and multivariate analysis for predictors of choledocholithiasis in acute calculous cholecystitis

\begin{tabular}{|c|c|c|c|c|}
\hline \multirow[t]{2}{*}{ Parameters } & \multicolumn{2}{|c|}{ Univariate } & \multicolumn{2}{|c|}{ Multivariate } \\
\hline & n $(\%)$ & $\mathrm{P}$-value & AOR $(95 \% \mathrm{CI})$ & P-value \\
\hline \multicolumn{5}{|c|}{ Age $>55$ years } \\
\hline Yes & $29 / 69$ & 0.211 & & \\
\hline No & $34 / 104$ & & & \\
\hline \multicolumn{5}{|l|}{ Sex } \\
\hline Male & $36 / 104$ & 0.546 & & \\
\hline Female & $27 / 69$ & & & \\
\hline \multicolumn{5}{|c|}{ Bilirubin $>1.8 \mathrm{mg} / \mathrm{dl}$} \\
\hline Yes & $42 / 94$ & 0.014 & & \\
\hline No & $21 / 79$ & & & \\
\hline \multicolumn{5}{|c|}{ ALT $>3 \mathrm{x}$ ULN } \\
\hline Yes & $18 / 45$ & 0.561 & & \\
\hline No & $45 / 128$ & & & \\
\hline \multicolumn{5}{|l|}{ ALP $>$ ULN } \\
\hline Yes & $55 / 124$ & 0.001 & $4.26(1.66-10.96)$ & 0.003 \\
\hline No & $8 / 49$ & & & \\
\hline \multicolumn{5}{|c|}{ Dilated CBD on USG } \\
\hline Yes & $47 / 72$ & 0.001 & $9.97(4.65-21.36)$ & 0.001 \\
\hline No & $16 / 101$ & & & \\
\hline \multicolumn{5}{|c|}{ CBDS on USG } \\
\hline Yes & $24 / 36$ & 0.001 & & \\
\hline No & $39 / 137$ & & & \\
\hline \multicolumn{5}{|c|}{ Acute biliary pancreatitis } \\
\hline Yes & $10 / 43$ & 0.039 & $0.36(0.14-0.91)$ & 0.030 \\
\hline No & $53 / 130$ & & & \\
\hline
\end{tabular}

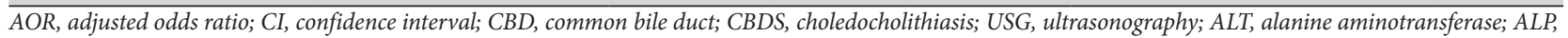
alkaline phosphatase; ULN, upper limit of normal

spontaneously [18]. In the APEC study of urgent ERCP in severe acute biliary pancreatitis, a total of $30.9 \%$ (71/230) patients had CBD stones or sludge in both study groups [20]. The remaining 69.1\% (159/230) did not have CBD stones, indicating that in a majority of patients a CBD stone passes the papilla spontaneously.

Taking the above into account, we propose a new algorithm, depicted in Fig. 1. Patients having score -1 or 0 should be directly referred for cholecystectomy. Those who have a score of 2 can be referred for preoperative ERCP or an intraoperative cholangiogram. Patients with a score of 1 should undergo endoscopic US or MRCP for confirmation of the CBD stone. Using this approach, $5 \%$ of patients will undergo cholecystectomy despite choledocholithiasis, an acceptable rate as per the previous literature [21]. However, 29\% of patients are at risk of undergoing diagnostic ERCP, slightly higher than the acceptable rate of $25 \%$ [22,23]. Using the ESGE and ASGE high likelihood criteria, the proportion of patients at risk of undergoing diagnostic ERCP is $31.1 \%$ and $31.6 \%$, respectively.

Chisholm et al developed a model using an increase in alanine aminotransferase to more than 3 times the upper limit of normal, raised ALP more than the upper limit of normal and dilated CBD on US. The authors showed that the presence of 0 or 1 risk factors rules out CBD stones in $98.6 \%$ of cases, and when the score was $3,77.8 \%$ of patients with ACC had choledocholithiasis [23]. This earlier predictive model appears better than the current study in the validation cohort. However, it was based on the odds ratio calculated from the univariate analysis [23] and did not evaluate the independence of each predictive factor by multivariate analysis.

Apart from being a retrospective study, the current study had a few other limitations. Our patients with ACC included a higher proportion of patients with coexisting choledocholithiasis (36\%) and acute biliary pancreatitis (25\%) [15]. This could be due to referral bias at a highly specialized endoscopic center. However, the suboptimal performance of current guidelines for predicting choledocholithiasis in ACC and the proposed model for evaluation can be utilized in general gastroenterology practice. We have used parameters for analysis at admission and have not studied the utility of serial LFT monitoring. However, previous studies have shown conflicting results regarding the role of serial LFT monitoring $[18,24,25]$ and the current guidelines do not recommend it.

To conclude, a significant proportion of patients with ACC have concomitant choledocholithiasis. The performance of the ASGE and ESGE guidelines' risk stratification criteria is inadequate in patients with ACC. We suggest the utilization of a separate predictive model for suspected choledocholithiasis in these patients. However, these findings need to be validated prospectively in the future. 


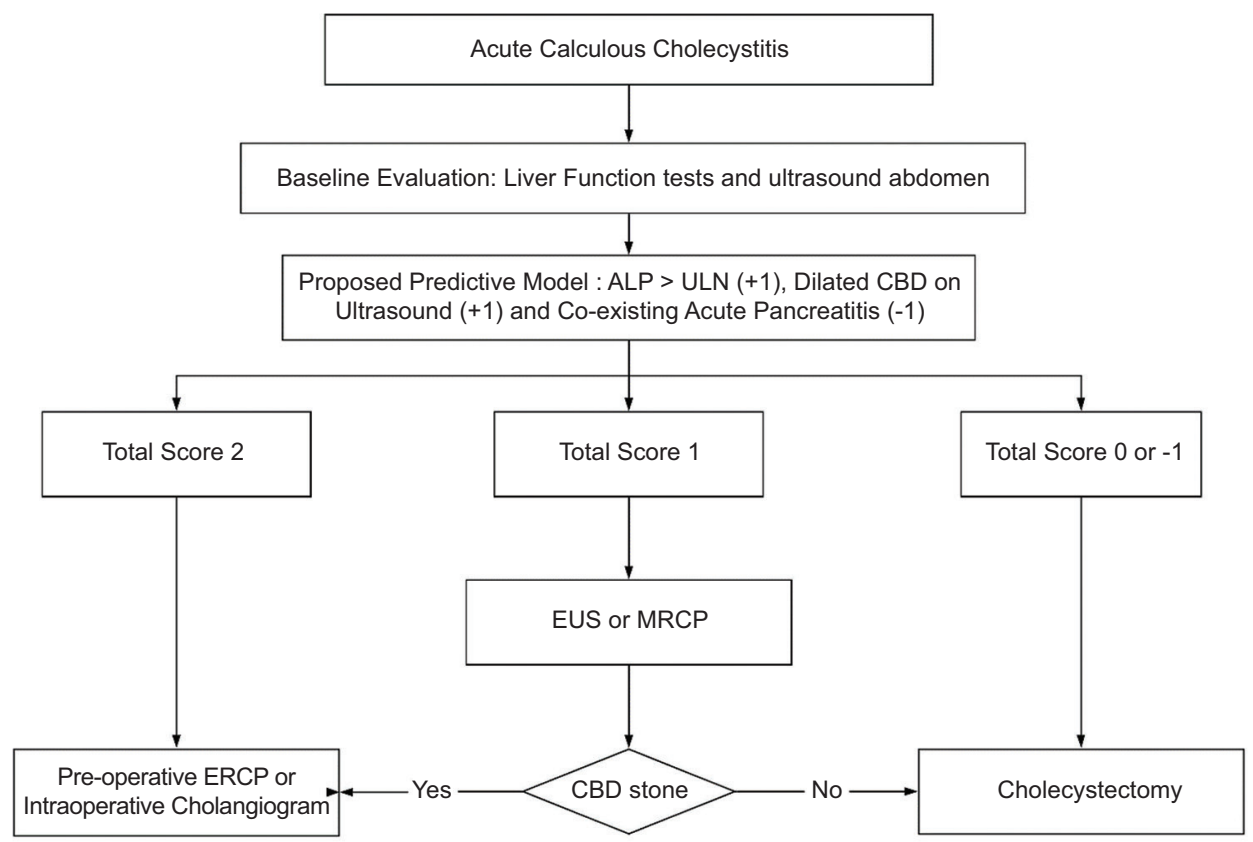

Figure 1 A proposed predictive model for choledocholithiasis in acute calculous cholecystitis ALP, alkaline phosphatase; ULN, upper limit of normal; CBD, common bile duct; ERCP, endoscopic retrograde cholangiopancreatography; EUS, endoscopic ultrasound

\section{Summary Box}

\section{What is already known:}

- Patients with acute calculous cholecystitis (ACC) can have coexisting choledocholithiasis

- Current guidelines for the evaluation of choledocholithiasis do not mention its application in patients with ACC

\section{What the new findings are:}

- ASGE and ESGE risk stratification algorithms are suboptimal in predicting choledocholithiasis in patients with ACC

- A separate predictive model can be used for the prediction of choledocholithiasis in ACC

\section{References}

1. Gomes CA, Junior CS, Di Saverio S, et al. Acute calculous cholecystitis: review of current best practices. World J Gastrointest Surg 2017;9:118-126.

2. Orlando $\mathrm{R}^{\text {rd }}$, Russell JC, Lynch J, Mattie A. Laparoscopic cholecystectomy. A statewide experience. The Connecticut Laparoscopic Cholecystectomy Registry. Arch Surg 1993;128: 494-498.

3. Riall TS, Zhang D, Townsend CM, Jr., Kuo YF, Goodwin JS. Failure to perform cholecystectomy for acute cholecystitis in elderly patients is associated with increased morbidity, mortality, and cost. J Am Coll Surg 2010;210:668-677, 677-679.

4. Strasberg SM. Clinical practice. Acute calculous cholecystitis. $N$ Engl J Med 2008;358:2804-2811.

5. Yokoe M, Hata J, Takada T, et al. Tokyo Guidelines 2018: diagnostic criteria and severity grading of acute cholecystitis (with videos). $J$ Hepatobiliary Pancreat Sci 2018;25:41-54.

6. Chen $\mathrm{H}$, Jorissen $\mathrm{R}$, Walcott J, Nikfarjam M. Incidence and predictors of common bile duct stones in patients with acute cholecystitis: a systematic literature review and meta-analysis. ANZ J Surg 2020;90:1598-1603.

7. Buxbaum JL, Abbas Fehmi SM, Sultan S, et al; ASGE Standards of Practice Committee. ASGE guideline on the role of endoscopy in the evaluation and management of choledocholithiasis. Gastrointest Endosc 2019;89:1075-1105.e15.

8. Manes G, Paspatis G, Aabakken L, et al. Endoscopic management of common bile duct stones: European Society of Gastrointestinal Endoscopy (ESGE) guideline. Endoscopy 2019;51:472-491.

9. Takada T, Strasberg SM, Solomkin JS, et al; Tokyo Guidelines Revision Committee. TG13: Updated Tokyo Guidelines for the management of acute cholangitis and cholecystitis. J Hepatobiliary Pancreat Sci 2013;20:1-7.

10. Banks PA, Bollen TL, Dervenis C, et al; Acute Pancreatitis Classification Working Group. Classification of acute pancreatitis--2012: revision of the Atlanta classification and definitions by international consensus. Gut 2013;62:102-111.

11. Jagtap N, Hs Y, Tandan M, et al. Clinical utility of ESGE and ASGE guidelines for prediction of suspected choledocholithiasis in patients undergoing cholecystectomy. Endoscopy 2020;52:569-573.

12. Geraghty JM, Goldin RD. Liver changes associated with cholecystitis. J Clin Pathol 1994;47:457-460.

13. Song SH, Kwon CI, Jin SM, et al. Clinical characteristics of acute cholecystitis with elevated liver enzymes not associated with choledocholithiasis. Eur J Gastroenterol Hepatol 2014;26:452-457. 
14. Tetangco EP, Shah N, Arshad HM, Raddawi H. Markedly elevated liver enzymes in choledocholithiasis in the absence of hepatocellular disease: case series and Literature review. I Investig Med High Impact Case Rep 2016;4:2324709616651092.

15. Padda MS, Singh S, Tang SJ, Rockey DC. Liver test patterns in patients with acute calculous cholecystitis and/or choledocholithiasis. Aliment Pharmacol Ther 2009;29:1011-1018.

16. Peng WK, Sheikh Z, Paterson-Brown S, Nixon SJ. Role of liver function tests in predicting common bile duct stones in acute calculous cholecystitis. Br J Surg 2005;92:1241-1247.

17. Ahn KS, Yoon YS, Han HS, Cho JY. Use of liver function tests as first-line diagnostic tools for predicting common bile duct stones in acute cholecystitis patients. World J Surg 2016;40:1925-1931.

18. He H, Tan C, Wu J, et al. Accuracy of ASGE high-risk criteria in the evaluation of patients with suspected common bile duct stones. Gastrointest Endosc 2017;86:525-532.

19. Gomez Hinojosa P, Espinoza-Rios J, Bellido Caparo A, et al. Accuracy of ASGE predictors in diagnosis of coledocolithiasis in a public hospital of Lima, Peru. Rev Gastroenterol Peru 2018;38:22-28.

20. Schepers NJ, Hallensleben NDL, Besselink MG, et al. Urgent endoscopic retrograde cholangiopancreatography with sphincterotomy versus conservative treatment in predicted severe acute gallstone pancreatitis (APEC): a multicentre randomized controlled trial. Lancet 2020;396:167-176.

21. Lee DH, Ahn YJ, Lee HW, Chung JK, Jung IM. Prevalence and characteristics of clinically significant retained common bile duct stones after laparoscopic cholecystectomy for symptomatic cholelithiasis. Ann Surg Treat Res 2016;91:239-246.

22. Elmunzer BJ, Debenedet AT, Volk ML, et al. Clinical yield of diagnostic endoscopic retrograde cholangiopancreatography in orthotopic liver transplant recipients with suspected biliary complications. Liver Transpl 2012;18:1479-1484.

23. Chisholm PR, Patel AH, Law RJ, et al. Preoperative predictors of choledocholithiasis in patients presenting with acute calculous cholecystitis. Gastrointest Endosc 2019;89:977-983.e2.

24. Adams MA, Hosmer AE, Wamsteker EJ, et al. Predicting the likelihood of a persistent bile duct stone in patients with suspected choledocholithiasis: accuracy of existing guidelines and the impact of laboratory trends. Gastrointest Endosc 2015;82:88-93.

25. Rubin MI, Thosani NC, Tanikella R, Wolf DS, Fallon MB, Lukens FJ. Endoscopic retrograde cholangiopancreatography for suspected choledocholithiasis: testing the current guidelines. Dig Liver Dis 2013;45:744-749. 